\title{
A revisit to the region of Collinder 132 using Carte du Ciel and Astrographic Catalogue plates (Corrigendum)
}

\author{
R. B. Orellana ${ }^{1}$, M. S. De Biasi ${ }^{1}$, I. H. Bustos Fierro ${ }^{2}$, and J. H. Calderón ${ }^{2}$ \\ ${ }^{1}$ Facultad de Ciencias Astronómicas y Geofisicas, UNLP, Paseo del Bosque s/n, 1900 La Plata \\ and Instituto de Astrofisica de La Plata (CCT La Plata - CONICET, UNLP), Argentina \\ e-mail: [rorellan; debiasi]@fcaglp.unlp.edu.ar \\ 2 Observatorio Astronómico, Laprida 854, 5000 Córdoba, Argentina \\ e-mail: [ivanbf; calderon]@mail.oac.uncor .edu
}

A\&A 521, A39 (2010), DOI: 10.1051/0004-6361/200913741

Key words. open clusters and associations: general - open clusters and associations: individual: Cr132 - astrometry - errata, addenda

A reference to Giuppone, C. A., Calderón, J. H., Bustos Fierro, I. H. 2006 work was inadvertently omitted in Sect. 2.2 and in the captions of figures therein, hence the following corrections should be introduced to the paper:

1. In Sect. 2.2, the last sentence of the first paragraph should read "The median of the internal errors in the proper motions is shown in Table 2 (as determined by Giuppone et al. 2006)".

2. In Sect. 2.2, the last sentence of the fourth paragraph should read "As also found by Giuppone et al. (2006), the internal errors derived for the proper motions were consistent with those resulting from the comparison with external catalogues, as shown in Table 3".

3. The caption of Fig. 3 should read "Residuals in both equatorial coordinates obtained using the individual reduction method. (Giuppone et al. 2006)".
4. The caption of Fig. 4 should read "Distribution of internal errors for stars with proper motions from two epochs (left) and from three epochs (right) (Giuppone et al. 2006)".

5. In Sect. 2.2, the first sentence of the last paragraph should read "The CCAC catalogue was constructed with 9055 mean stellar positions on the International Celestial Reference System (ICRS) with their corresponding epochs in the selected field, including the components of proper motion with their errors for only 8774 stars (Giuppone et al. 2006)".

6. The following reference should be added:

\section{References}

Giuppone, C. A., Calderón, J. H., \& Bustos Fierro, I. H. 2006, Trabajo Especial de Licenciatura en Astronomía, Facultad de Matemática, Astronomía y Física, Universidad Nacional de Córdoba 